Nota científica

(Short communication)

\title{
REGISTRO NOTABLE DE LA GOLONDRINA PUEBLERA PETROCHELIDON FULVA (VIEILLOT, 1808): PASSERIFORMES, HIRUNDINIDAE, EN EL ESTADO DE OAXACA, MÉXICO
}

\author{
NOTEWORTHY RECORD OF THE CAVE SWALLOW PETROCHELIDON FULVA \\ (VIEILLOT, 1808): PASSERIFORMES, HIRUNDINIDAE, IN THE STATE OF OAXACA, \\ MEXICO
}

\author{
ELDER RUIZ VELÁSQUEZ*, ANTONIO SANTOS MORENO \\ Laboratorio de Ecología Animal. Centro Interdisciplinario de Investigación para el Desarrollo Integral Regional, Unidad Oaxaca, \\ Instituto Politécnico Nacional. Calle Hornos No. 1003, Santa Cruz Xoxocotlán, Oaxaca, Código Postal 71230. \\ <elder.rv@hotmail.com>; <asantosm90@hotmail.com> \\ *Autor de correspondencia: <elder.rv@hotmail.com> \\ Recibido: 01/09/2017; aceptado: 23/08/2019; publicado en línea: 12/09/2019 \\ Editor responsable: Ricardo Rodríguez Estrella
}

Ruiz-Velásquez, E., Santos-Moreno, A. (2019) Registro notable de la golondrina Petrochelidon fulva (Vieillot, 1808): Passeriformes, Hirundinidae en el estado de Oaxaca, México. Acta Zoológica Mexicana (nueva serie), 35, 1-4. https://doi.org/10.21829/azm.2019.3502238

RESUMEN. En este trabajo se documenta por primera vez la presencia de la golondrina pueblera (Petrochelidon fulva) en la región del Balsas de Oaxaca, México, así como su actividad reproductiva en la entidad. Con este registro se documenta la expansión de la golondrina pueblera en el Estado.

Palabras clave: Petrochelidon fulva; Oaxaca; expansión de área; registro

Ruiz-Velásquez, E., Santos-Moreno, A. (2019) Noteworthy record of the cave swallow Petrochelidon fulva (Vieillot, 1808): Passeriformes, Hirundinidae in the state of Oaxaca, Mexico. Acta Zoológica Mexicana (nueva serie), 35, 1-4. https://doi.org/10.21829/azm.2019.3502238

ABSTRACT. In this paper, we document for first time the presence of the Cave Sallow (Petrochelidon fulva) in the Region of the Balsas in the state of Oaxaca, southeastern Mexico, as well as the establishment of reproductive colonies in the state.

Key words: Petrochelidon fulva; Oaxaca; expansion area; record

Aunque la exploración ornitológica en el estado de Oaxaca comenzó hace cerca ya de dos siglos (Binford, 1989), amplias porciones de las regiones avifaunísticas Cuenca del Balsas y el Eje Neovolcánico Transversal ubicadas en Oaxaca han sido poco exploradas. Estas regiones cuentan con menos de 100 especies, pero es relevante que la Cuenca del Balsas cuenta con el mayor número de endemismos en el Estado (Navarro et al., 2004). En México la familia Hirundinidae (Orden Passeriformes) está representada 
por ocho géneros y 13 especies, 10 de ellas con presencia confirmada en el estado de Oaxaca (Navarro et al., 2004; Berlanga et al., 2015). En Oaxaca, el género Petrochelidon está representado por dos especies, $P$. fulva y $P$. pyrrhonota, a $P$. fulva solo se le ha registrado en la región del Istmo (McAndrewa, 2019) mientras que $P$. pyrrhonota se distribuye en las regiones avifaunísticas del Pacífico, Sierra Sur, Istmo y Atlántico (Navarro et al., 2004).

Petrochelidon pyrrhonota presenta una rabadilla de color rojizo o ante. En vuelo da la impresión de que la forma de la cola es cuadrada y se denota un parche obscuro en la garganta. Planea volando arriba y abajo, terminando cada planeo elevándose en una pendiente más pronunciada que las otras golondrinas. $P$. fulva es parecida a $P$. pyrrhonota, pero con la garganta clara y la frente castaño obscuro (Peterson \& Chalif, 2000). En $P$. fulva la frente es de color rojo ladrillo. Por otro lado, a pesar de que algunos individuos de $P$. pyrrhonota pueden tener la frente de color canela, en general la tienen de color ante o rufo blanquecino. Además, $P$. fulva presenta la coloración ante o rufo en la parte superior del pecho y flancos más marcada y extendida que $P$. pyrhronota. La cola ligeramente ahorquillada de $P$. fulva la diferencía de $P$. pyrrhonota, que la presenta cuadrada (Davis, 2005). En México, existen tres zonas bien definidas de distribución de $P$. fulva: la Península de Yucatán, el centro de Chiapas y el Altiplano Mexicano.

El día 5 de abril de 2016 observamos ejemplares adultos de $P$. fulva y nidos adheridos por debajo del puente de la carretera estatal número 34 Huajuapan de León-Juxtlahuaca (17 $51^{\circ} 43.55^{\prime \prime}$ N, 98 9 8' 56.52" W) en el municipio de Mariscala de Juárez, a una altitud de $1,062 \mathrm{~m}$, en el noroeste del estado de Oaxaca (Fig. 1). En la zona, la temperatura varía de $\operatorname{los} 18$ a $\operatorname{los} 26^{\circ} \mathrm{C}$, con una precipitación que fluctúa entre los 700 y los $1,000 \mathrm{~mm}$, el clima es cálido subhúmedo con lluvias en verano. En esta área predomina la selva baja caducifolia y las especies dominantes son Bursera copallifera, B. schechtendali, Lysiloma divaricata y L. acapulcensis. Toda la región es atravesada por el río Mixteco, afluente del río Balsas. El punto más cercano del área de registro se ubica a $369 \mathrm{~km}$ en La Venta, Juchitán, también en Oaxaca (McAndrews, 2019) (Fig. 1).

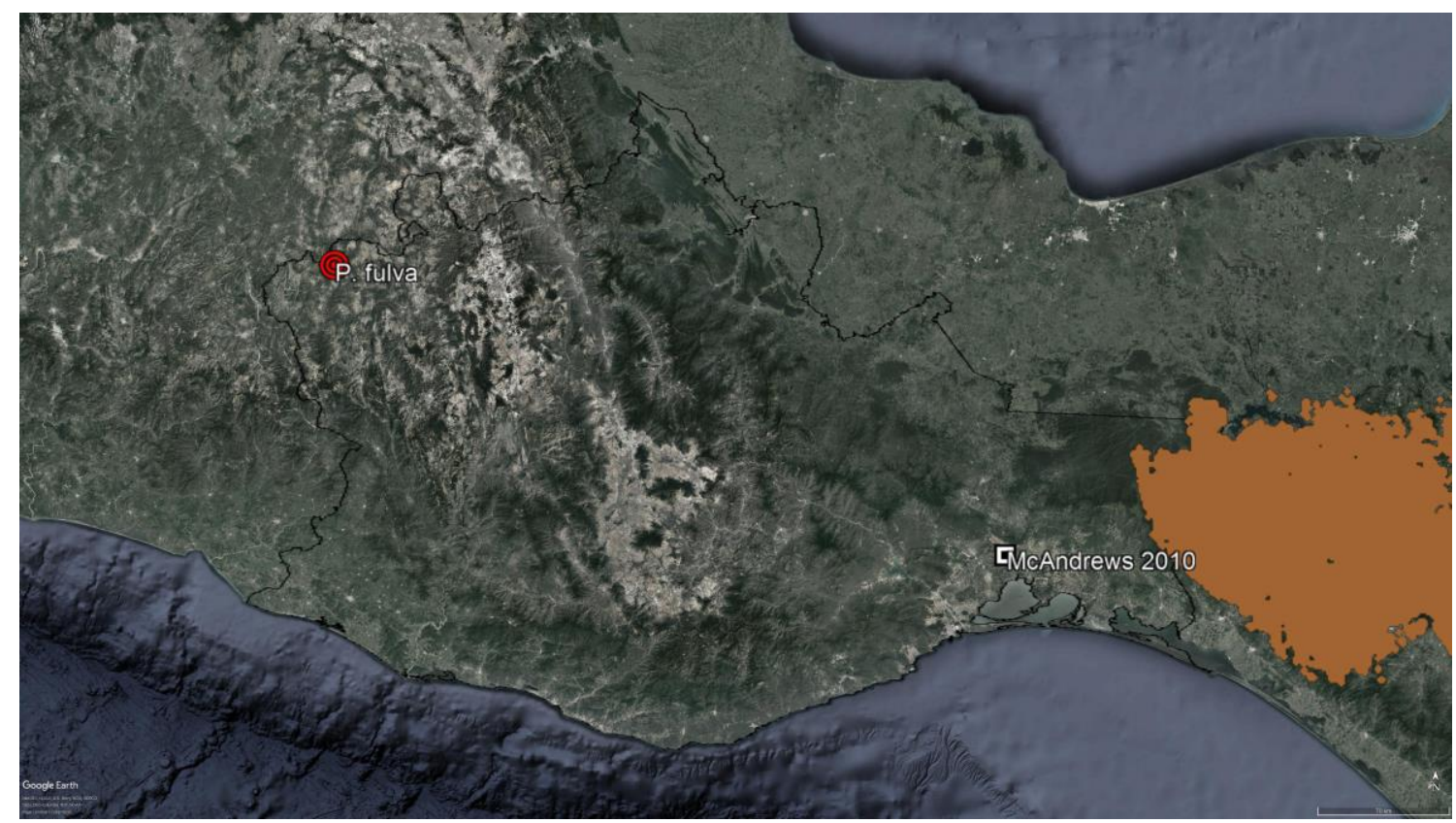

Figura 1. Distribución potencial de la golondrina pueblera (Petrochelidon fulva) se denota en color café (Navarro \& Peterson, 2007). Se muestra nuestro registro confirmado de la especie en Oaxaca (círculo rojo) y el registro previo más cercano (cuadro blanco). 
Se registraron 18 individuos adultos y 41 nidos ubicados en posición continua entre sí, con forma de copa, hechos de lodo y en 25 de los nidos se observaron crías de $P$. fulva (Fig. 2). De los nidos registrados, uno estaba ocupado por el Pinzón mexicano (Haemorhous mexicanus), otro por el papamoscas cardenalito (Pyrocephalus rubinus) y dos por el gorrión doméstico (Passer domesticus). Cuando no estaban en los nidos, los individuos de $P$. fulva mostraron actividad de vuelo rasante sobre el río, lo que evidenció que se alimentaban. En los sobrevuelos en el río, P. fulva interactuó con la golondrina ala serrada (Stelgidopteryx serripennis).

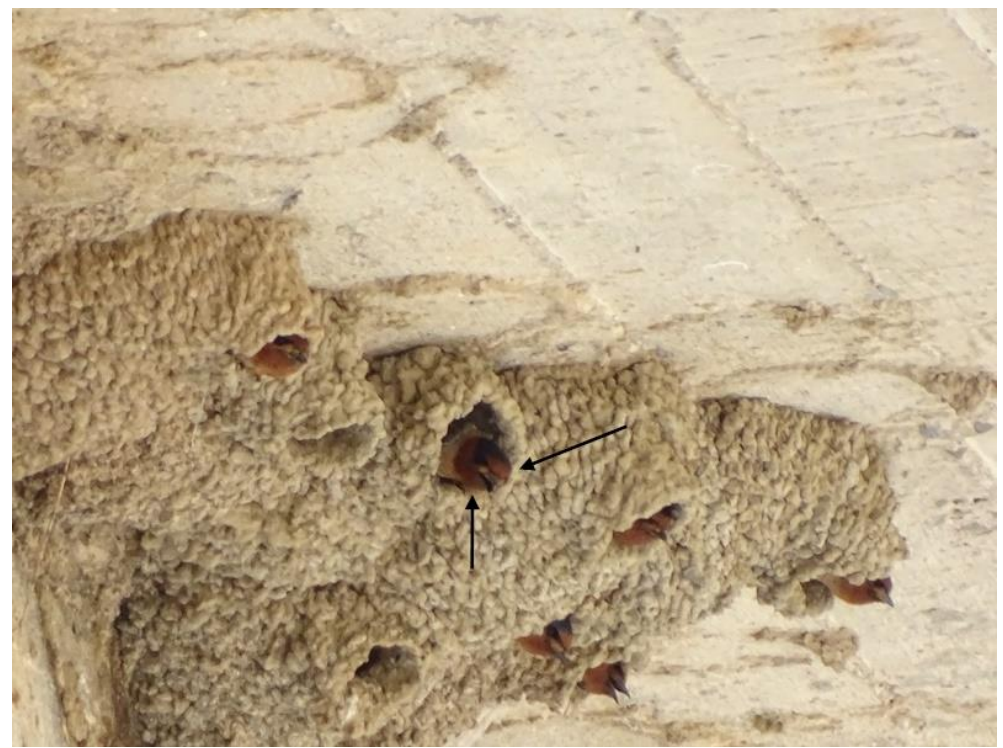

Figura 2. Nidos de la golondrina pueblera Petrochelidon fulva debajo del puente del río Mixteco, Oaxaca, México. Nótense los caracteres distintivos de la especie (flechas).

Ya se tenían registros previos de $P$. fulva en cuatro localidades del estado de Oaxaca, La Ventosa, Unión Hidalgo, Santo Domingo Ingenio y Santa María Xadani (Avibase, 2019), pero el presente registro documenta por primera vez la ocurrencia de la golondrina pueblera en la Región del Balsas en el estado Oaxaca, y también por primera vez se documenta su actividad reproductiva en la entidad. El registro aquí documentado ratifica la tendencia de expansión de la especie, aunque es en la porción norteña de su distribución donde $P$. fulva ha tenido una de las expansiones más dramáticas. En los Estados Unidos de Norteamérica era poco común en la década de 1960 (National Audubon Society, 2014), pero entre 1957 y 1999 ha incrementado la extensión de su zona de crianza en un $898 \%$ aproximadamente, con un incremento promedio anual de 6\%; se vaticina que probablemente continuará esta expansión (Kosciuch et al., 2006).

Se ha observado que la expansión y colonización de nuevos sitios se debe a la presencia de nuevos refugios para esta especie, tales como la presencia de puentes, bóvedas, losas, y otras estructuras que se emplean en la construcción de carreteras donde existen escorrentías, principalmente en las carreteras (Martin \& Martin, 1978). En el caso de nuestra área de registro, es probable que el incremento en el número de puentes donde corre el agua del río Balsas sea un factor que beneficia a la especie y promueve la expansión de sus poblaciones. Por otro lado, el impacto de la expansión de $P$. fulva en otras golondrinas es desconocido, por lo que se sugiere dar seguimiento a las poblaciones de la especie y evaluar su efecto en otras especies de golondrinas.

Agradecimientos. Agradecemos al Dr. José Roberto Sosa López y el M. en C. Wiliam Ku Peralta del Laboratorio de Bioacústica y Ecología del Comportamiento del CIIDIR-Oaxaca que ayudaron a la 
corroboración de la identificación de la especie. El Instituto Politécnico Nacional de México proporcionó apoyo financiero.

\section{LITERATURA CITADA}

Avibase. The World Birds Database (2019) Disponible en: https://avibase.bsceoc.org/species.jsp?lang=EN\&avibaseid=79A1EDEC9657AA3C\&sec=ebird (consultado el 13 de junio de 2019).

Berlanga, H., Gómez de Silva, H., Vargas-Canales, V. M., Rodríguez-Contreras, V., SánchezGonzález, L. A., Ortega-Álvarez, R., Calderón-Parra, R. (2015) Aves de México: Lista actualizada de especies y nombres comunes. CONABIO, México D.F. 117 pp.

Binford, L. C. (1989) A distributional survey of the birds of the Mexican state of Oaxaca. Ornithological Monographs, 43, 1-428.

Davis, P. (2005) Maryland / District of Columbian Records Committee. Skins workshops March 2005. Disponible en: www.mdbirds.org/mddcrc/pdf/rcskins2005.pdf

Kosciuch, K. L., Ormston, C. G., Arnold, K. A. (2006) Breeding Range Expansion by Cave Swallows (Petrochelidon fulva) in Texas. The Southwestern Naturalist, 51 (2), 203-209.

Martin, R. F., Martin, S. R. (1978) Niche and range expansion of cave swallows in Texas. American Birds, 32, 941-946.

McAndrewa, A. (2019) Registro de Golondrina pueblera/Petrochelidon fulva. eBird: a citizen-based bird observation network in the biological sciences. Disponible en: https://ebird.org/averaves/species/cavswa/MX-OAX

National Audubon Society (2014) Guía de Aves de América del Norte. Golondrina PuebleraPetrochelidon fulva. Disponible en: http://www.audubon.org/es/guia-de-aves/ave/golondrinapueblera

Navarro, S. A. G., García-Trejo, E. A., Peterson, A. T., Rodríguez-Contreras, V. (2004) Aves, pp. 391421. En: García-Mendoza, A. J., Ordóñez, M. J., Briones-Salas, M. (Eds.). Biodiversidad de Oaxaca. Instituto de Biología, UNAM-Fondo Oaxaqueño para la Conservación de la NaturalezaWorld Wildlife Fund, México.

Peterson, R. T., Chalif, E. L. (2000) Aves de México guía de campo, Identificación de todas las especies encontradas en México, Guatemala, Belice y El Salvador. Editorial Diana, México. 473 pp. 